\title{
Explicit Travelling Wave Solutions to Nonlinear Partial Differential Equations Arise in Mathematical Physics and Engineering
}

\author{
Muktarebatul Jannah, Md. Tarikul Islam*, Mst. Armina Akter \\ Department of Mathematics, Hajee Mohammad Danesh Science and Technology University, Dinajpur, Bangladesh
}

Received: January 23, 2021, Revised: March 11, 2021, Accepted: March 13, 2021, Available Online: March 30, 2021

\begin{abstract}
To describe the interior phenomena of the mysterious problems around the real world, non-linear partial differential equations (NLPDEs) plays a substantial role, for which construction of analytic solutions of those is most important. This paper stands for a goal to find fresh and wide-ranging solutions to some familiar NLPDEs namely the non-linear cubic Klein-Gordon (cKG) equation and the non-linear Benjamin-Ono (BO) equation. A wave variable transformation is made use to convert the mentioned equations into ordinary differential equations. To acquire the desired precise exact travelling wave solutions to the above-stated equations, the rational $\left(G^{\prime} / G\right)$ expansion method is employed. Consequently, three types of equipped solutions are successfully come out in the forms of hyperbolic, trigonometric and rational functions in a compatible way. To analyse the physical problems arisen relating to nonlinear complex dynamical systems, our obtained solutions might be most helpful. So far we know, these achieved solutions are different than those in the literature. The applied method is efficient and reliable which might further be used to find different and novel solutions to many other NLPDEs successfully in research field.
\end{abstract}

Keywords: The rational $\left(G^{\prime} / G\right)$-expansion method, nonlinear partial differential equation, complex transformation, exact solution.

This work is licensed under a Creative Commons Attribution-Non Commercial 4.0 International License.

\section{Introduction}

In this new era, nonlinear phenomena have arisen in a wide range in the area of extensive physical science and mathematics. The nonlinear mechanism of nature can be depicted by NLPDEs. For this reason, with the rapid development of nonlinear sciences, it has debuted with a lot of importance in physical science and mathematics and many research works have been done to analyse these equations. A special case, the closed form solutions of NLPDEs bears significant role to delineate many models concerning the underlying mechanisms of real world. Subsequently, research on this topic is becoming as a matter of attraction in the field of nonlinear science day by day. With this importance, for the analytical solutions of NLPDEs, many traditional techniques have been emerged and implemented to solve various kinds of problems such as the Adomian decomposition method [1]-[2], the tanh function method [3]-[4], the simplest equation method [5]-[6], the Jacobi elliptic function method [7]-[8], the expansion function method [9]-[10], the modified simple equation method [11]-[12], the $\left(G^{\prime} / G\right)$ expansion method [13]-[14], the cosh function method [15]-[16], the homotopy perturbation method [17]-[18], the multiple expfunction method [19]-[20], the Bernoulli sub-ODE method [21][22], the homotopy analysis method [23]-[24], the variational iteration method [25]-[26], the modified tanh-coth method and the extended Jacobi elliptic function method [27], the $\mathrm{He}$ homotopy perturbation method [28]-[29], the homogeneous balance method [30], the inverse scattering method [31], the Backlund transformation method [32]-[33], the extended modified direct algebraic method [34]-[37]. In this paper, we have described the rational $\left(G^{\prime} / G\right)$-expansion method [38]-[41].

\section{Explanation of the Technique}

Consider the following NLPDE:
$F\left(u, u_{x}, u_{t}, u_{x x}, u_{t t}, u_{t x} \cdots\right)=0$

where $u=u(x, t)$ and the subscripts in $u$ represents partial derivatives. Followings are the main steps of the rational $\left(G^{\prime} / G\right)$-expansion method:

First step: Introduce the transformation

$$
u(x, t)=U(\xi), \xi=x \pm v t
$$

where $v$ stands for the wave velocity. This transformation reduces Eq. (1) to the ordinary differential equation with respect to $\xi$,

$$
Q\left(U, U^{\prime}, U^{\prime \prime}, U^{\prime \prime \prime}, \cdots\right)=0
$$

Second step: Take anti-derivative of Eq. (3) as much as possible; the integral constant may be considered as zero for seeking solitary wave solutions.

Third step: Consider the solution of Eq. (3) as follows:

$$
U(\xi)=\frac{\sum_{i=0}^{n} a_{i}\left(G^{\prime} / G\right)^{i}}{\sum_{i=0}^{n} b_{i}\left(G^{\prime} / G\right)^{i}}
$$

with unknown constants $a_{i}$ and $b_{i}(i=0,1,2, \cdots, n)$ in which at least one of $a_{n}$ and $b_{n}$ is non-zero. $G=G(\xi)$ satisfies the ordinary differential equation,

$$
G^{\prime \prime}(\xi)+\lambda G^{\prime}(\xi)+\mu G(\xi)=0
$$

where $\lambda$ and $\mu$ are real parameters. Eq. (5) has turned into

$$
\frac{d}{d \xi}\left(G^{\prime} / G\right)=-\left(G^{\prime} / G\right)^{2}-\lambda\left(G^{\prime} / G\right)-\mu
$$

Then we have the general solutions of Eq. (5) (or equivalent to Eq. (6)) as follows: 
$\left(G^{\prime} / G\right)=-\frac{\lambda}{2}+$

$\frac{\sqrt{\lambda^{2}-4 \mu}}{2}\left(\frac{A \sinh \left(\left(\sqrt{\lambda^{2}-4 \mu} / 2\right) \xi\right)+B \cosh \left(\left(\sqrt{\lambda^{2}-4 \mu} / 2\right) \xi\right)}{A \cosh \left(\left(\sqrt{\lambda^{2}-4 \mu} / 2\right) \xi\right)+B \sinh \left(\left(\sqrt{\lambda^{2}-4 \mu} / 2\right) \xi\right)}\right), \lambda^{2}-$

$4 \mu>0$

$\left(G^{\prime} / G\right)=-\frac{\lambda}{2}+$

$\frac{\sqrt{4 \mu-\lambda^{2}}}{2}\left(\frac{-A \sin \left(\left(\sqrt{4 \mu-\lambda^{2}} / 2\right) \xi\right)+B \cos \left(\left(\sqrt{4 \mu-\lambda^{2}} / 2\right) \xi\right)}{A \cos \left(\left(\sqrt{4 \mu-\lambda^{2}} / 2\right) \xi\right)+B \sin \left(\left(\sqrt{4 \mu-\lambda^{2}} / 2\right) \xi\right)}\right), \quad \lambda^{2}-$

$4 \mu<0$

$\left(G^{\prime} / G\right)=-\frac{\lambda}{2}+\frac{B}{A+B \xi}$,

$\lambda^{2}-4 \mu=0$

where $A$ and $B$ are real parameters.

Fourth step: Determine $n$ by applying homogeneous balance to Eq. (3) for the degree of $U(\xi)$ as $n$. Then

$$
\begin{aligned}
& \operatorname{deg}\left[\frac{d^{n} U(\xi)}{d \xi^{m}}\right]=n+m, \quad \operatorname{deg}\left[U^{m}\left(\frac{d^{l} U(\xi)}{d \xi^{l}}\right)^{p}\right]=m n+ \\
& p(n+l) .
\end{aligned}
$$

Fifth step: Eq. (3) with Eqs. (4), (5) makes a polynomial $\left(G^{\prime} / G\right)$. Set each coefficient to zero and solve them by the computer software Maple to calculate the values of $a_{i}, b_{i}, \mu$ and $v$.

Sixth step: Inserting the values determined in fifth step along with the outcomes given in Eqs. (7)-(9) into solution Eq. (4) provides the solutions of Eq. (1).

\section{Implementation of the Technique}

Herein, the suggested scheme is applied to examine the exact analytic solutions to the considered equations.

\subsection{The $\mathrm{cKG}$ equation}

The $\mathrm{cKG}$ equation is

$$
u_{x x}+u_{y y}-u_{t t}+a u+b u^{3}=0
$$

The wave variable transformation $u(x, y, t)=U(\xi), \xi=x+$ $y-c t$ reduces Eq. (10) into the following equation:

$\left(2-c^{2}\right) U^{\prime \prime}+a U+b U^{3}=0$

Due to homogeneous balance method, Eq. (11) gives $n=1$ and Eq. (4) turns into the form

$U(\xi)=\frac{a_{0}+a_{1}\left(G^{\prime} / G\right)}{b_{0}+b_{1}\left(G^{\prime} / G\right)}$

Inserting Eq. (12) into Eq. (11) provides a polynomial in $\left(G^{\prime} / G\right)$. Set the coefficients to zero and solved by computational software Maple. Accordingly, the following outcomes for $a_{0}, a_{1}, b_{0}, b_{1}$ and $c$ are obtained:

Set-1:

$$
\begin{aligned}
& a_{0}= \pm \frac{b_{1}}{2} \sqrt{\frac{a\left(-\lambda^{2}+4 \mu\right)}{b}}, \quad a_{1}=0, \quad b_{0}=\frac{b_{1} \lambda}{2}, \quad c= \\
& \pm \sqrt{\frac{2\left(a+4 \mu-\lambda^{2}\right)}{-\lambda^{2}+4 \mu}}
\end{aligned}
$$

Set-2:

$$
\begin{aligned}
& a_{0}=\mp\left(2 b_{1} \mu-\lambda b_{0}\right) \sqrt{\frac{a}{b\left(-\lambda^{2}+4 \mu\right)}}, \\
& a_{1}= \pm\left(2 b_{0}-b_{1} \lambda\right) \sqrt{\frac{a}{b\left(-\lambda^{2}+4 \mu\right)}}, \\
& c= \pm \sqrt{\frac{2\left(a+4 \mu-\lambda^{2}\right)}{\left(-\lambda^{2}+4 \mu\right)}}
\end{aligned}
$$

Set-3:

$$
\begin{aligned}
& a_{0}=\frac{\mathrm{b}_{1}\left(-2 \mu+\lambda\left(\frac{\lambda}{2} \pm \frac{1}{6} \sqrt{-12 \mu+3 \lambda^{2}}\right)\right) \sqrt{-3 \mathrm{ab}}}{\mathrm{b} \sqrt{-12 \mu+3 \lambda^{2}}} \\
& a_{1}= \pm \frac{\sqrt{-3 \mathrm{ab}}}{3 \mathrm{~b}} \mathrm{~b}_{1} \\
& b_{0}=\left(\frac{\lambda}{2} \pm \frac{1}{6} \sqrt{-12 \mu+3 \lambda^{2}}\right) b_{1} \\
& c= \pm \sqrt{\frac{2\left(a+4 \mu-\lambda^{2}\right)}{\left(-\lambda^{2}+4 \mu\right)}}
\end{aligned}
$$

Set-4:

$a_{0}=-\frac{\mathrm{b}_{1}\left(-2 \mu+\lambda\left(\frac{\lambda}{2} \pm \frac{1}{6} \sqrt{-12 \mu+3 \lambda^{2}}\right)\right) \sqrt{-3 \mathrm{ab}}}{\mathrm{b} \sqrt{-12 \mu+3 \lambda^{2}}}$,

$a_{1}= \pm \frac{\sqrt{-3 \mathrm{ab}}}{3 \mathrm{~b}} \mathrm{~b}_{1}$

$$
\begin{aligned}
& b_{0}=\left(\frac{\lambda}{2} \pm \frac{1}{6} \sqrt{-12 \mu+3 \lambda^{2}}\right) b_{1}, \\
& c= \pm \sqrt{\frac{2\left(a+4 \mu-\lambda^{2}\right)}{\left(-\lambda^{2}+4 \mu\right)}}
\end{aligned}
$$

Eq. (12) along with Eq. (13) becomes

$$
U_{1}(\xi)=\sqrt{\frac{a}{b}} \frac{\sqrt{-\lambda^{2}+4 \mu}}{\lambda+2\left(G^{\prime} / G\right)}
$$

Where,

$$
\xi=x+y \mp \sqrt{\frac{2\left(a+4 \mu-\lambda^{2}\right)}{-\lambda^{2}+4 \mu}} t .
$$

Utilizing the solutions in Eqs. (7)-(9) from Eq. (17), we make available the following solutions to Eq. (10) in the following three different forms:

Case 1: When $\lambda^{2}-4 \mu>0$,

Eq. (12) along with Eq. (13) becomes

$$
\begin{aligned}
& U_{1_{1}}(\xi) \\
& =i \sqrt{\frac{a}{b}} \frac{A \cosh \left(\left(\sqrt{\lambda^{2}-4 \mu} / 2\right) \xi\right)+B \sinh \left(\left(\sqrt{\lambda^{2}-4 \mu} / 2\right) \xi\right)}{A \sinh \left(\left(\sqrt{\lambda^{2}-4 \mu} / 2\right) \xi\right)+B \cosh \left(\left(\sqrt{\lambda^{2}-4 \mu} / 2\right) \xi\right)}
\end{aligned}
$$

where,

$$
\xi=x+y \mp \sqrt{\frac{2\left(a+4 \mu-\lambda^{2}\right)}{-\lambda^{2}+4 \mu}} t
$$


For $A=0$ and $B \neq 0$, we get

$$
U_{1_{2}}(\xi)=i \sqrt{\frac{a}{b}} \tanh \left(\left(\sqrt{\lambda^{2}-4 \mu} / 2\right) \xi\right)
$$

Assigning $A \neq 0$ and $B=0$ yields

$$
U_{1_{3}}(\xi)=i \sqrt{\frac{a}{b}} \operatorname{coth}\left(\left(\sqrt{\lambda^{2}-4 \mu} / 2\right) \xi\right)
$$

Case 2: For $\lambda^{2}-4 \mu<0$,

$$
U_{1_{4}}(\xi)=\sqrt{\frac{a}{b}} \frac{A \cos \left(\left(\sqrt{4 \mu-\lambda^{2}} / 2\right) \xi\right)+B \sin \left(\left(\sqrt{4 \mu-\lambda^{2}} / 2\right) \xi\right)}{-A \sin \left(\left(\sqrt{4 \mu-\lambda^{2}} / 2\right) \xi\right)+B \cos \left(\left(\sqrt{4 \mu-\lambda^{2}} / 2\right) \xi\right)}
$$

where,

$\xi=x+y \mp \sqrt{\frac{2\left(a+4 \mu-\lambda^{2}\right)}{-\lambda^{2}+4 \mu}} t$.

Appling $A=0$ and $B \neq 0$, provides

$$
U_{1_{5}}(\xi)=\sqrt{\frac{a}{b}} \tan \left(\left(\sqrt{4 \mu-\lambda^{2}} / 2\right) \xi\right)
$$

Using $A \neq 0$ and $B=0$ gives

$$
U_{1_{6}}(\xi)=-\sqrt{\frac{a}{b}} \cot \left(\left(\sqrt{4 \mu-\lambda^{2}} / 2\right) \xi\right)
$$

Case 3: If $\lambda^{2}-4 \mu=0$, the method yields stationary wave solutions and thus have not been documented.

Using the similar procedure for the all-other sets of solutions, as we have applied for set-1, we obtain the hyperbolic solutions for $\lambda^{2}-4 \mu>0$, the trigonometric solutions for $\lambda^{2}-4 \mu<0$, and $\lambda^{2}-4 \mu=0$ gives the stationary wave solutions.

\subsection{The BO equation}

Consider the BO equation

$$
u_{t}+h u_{x x}+u u_{x}=0
$$

Using the transformation $u(x, t)=U(\xi), \xi=x-c t$, Eq. (24) reduces to the ODE

$$
-c U^{\prime}+h U^{\prime \prime}+U U^{\prime}=0
$$

Integrating Eq. (25) gives

$$
r-c U+h U^{\prime}+\frac{1}{2} U^{2}=0,
$$

where $r$ is the integral constant. Appling homogeneous balance to $U^{2}$ and $U^{\prime}$ produces $n=1$ for which the solution (4) becomes

$$
U(\xi)=\frac{a_{0}+a_{1}\left(G^{\prime} / G\right)}{b_{0}+b_{1}\left(G^{\prime} / G\right)}
$$

where at least one of $a_{1}$ or $b_{1}$ is non zero.

Inserting Eq. (27) into Eq. (26) makes a polynomial in $\left(G^{\prime} / G\right)$. Setting the coefficients to zero and calculating by computer software Maple provides the following values for $a_{0}, a_{1}, b_{0}, b_{1}$ and $c$ :

$$
\begin{aligned}
& a_{0}=\frac{b_{1}}{2 h}\left\{(c+h \lambda)\left(c \pm \sqrt{c^{2}-2 r}\right)-2 r\right\}, \\
& a_{1}=b_{1}\left(c \pm \sqrt{c^{2}-2 r}\right), \\
& b_{0}=\frac{b_{1}}{2 h}\left(h \lambda \pm \sqrt{c^{2}-2 r}\right),
\end{aligned}
$$

Eq. (27) together with the values in Eq. (28) reduces to

$$
\begin{aligned}
& U(\xi) \\
& =\frac{\left\{(c+h \lambda)\left(c \pm \sqrt{c^{2}-2 r}\right)-2 r\right\}+2 h\left(c \pm \sqrt{c^{2}-2 r}\right)\left(G^{\prime} / G\right)}{\left(h \lambda \pm \sqrt{c^{2}-2 r}\right)+2 h\left(G^{\prime} / G\right)}
\end{aligned}
$$

where $\xi=x-c t$.

Eq. (29) with the aid of Eqs. (7)-(9) serves the following exact solutions to Eq. (24) in three types as hyperbolic, trigonometric and rational:

Case 1: If $\lambda^{2}-4 \mu>0$

$$
\begin{aligned}
& U_{1}(\xi) \\
& =\frac{+2 h\left(c \pm \sqrt{c^{2}-2 r}\right)\left(-\frac{\lambda}{2}+\frac{\sqrt{\lambda^{2}-4 \mu}}{2} \times \frac{A \sinh \left(\left(\sqrt{\lambda^{2}-4 \mu} / 2\right) \xi\right)+B \cosh \left(\left(\sqrt{\lambda^{2}-4 \mu} / 2\right) \xi\right)}{A \cosh \left(\left(\sqrt{\lambda^{2}-4 \mu} / 2\right) \xi\right)+B \sinh \left(\left(\sqrt{\lambda^{2}-4 \mu} / 2\right) \xi\right)}\right)}{\left(h \lambda \pm \sqrt{c^{2}-2 r}\right)+2 h\left(-\frac{\lambda}{2}+\frac{\sqrt{\lambda^{2}-4 \mu}}{2} \times \frac{A \sinh \left(\left(\sqrt{\lambda^{2}-4 \mu} / 2\right) \xi\right)+B \cosh \left(\left(\sqrt{\lambda^{2}-4 \mu} / 2\right) \xi\right)}{A \cosh \left(\left(\sqrt{\lambda^{2}-4 \mu} / 2\right) \xi\right)+B \sinh \left(\left(\sqrt{\lambda^{2}-4 \mu} / 2\right) \xi\right)}\right)}
\end{aligned}
$$

where $\xi=x-c t$.

If $A=0, B \neq 0$, then

$$
\begin{aligned}
& U_{1_{1}}(\xi) \quad\left\{(c+h \lambda)\left(c \pm \sqrt{c^{2}-2 r}\right)-2 r\right\} \\
& =\frac{+2 h\left(c \pm \sqrt{c^{2}-2 r}\right)\left(-\frac{\lambda}{2}+\frac{\sqrt{\lambda^{2}-4 \mu}}{2} \times \operatorname{coth}\left(\left(\sqrt{\lambda^{2}-4 \mu} / 2\right) \xi\right)\right)}{\left(h \lambda \pm \sqrt{c^{2}-2 r}\right)+2 h\left(-\frac{\lambda}{2}+\frac{\sqrt{\lambda^{2}-4 \mu}}{2} \times \operatorname{coth}\left(\left(\sqrt{\lambda^{2}-4 \mu} / 2\right) \xi\right)\right)}
\end{aligned}
$$

Again, for $A \neq 0, B=0$,

$$
\begin{aligned}
& U_{1_{2}}(\xi) \quad\left\{(c+h \lambda)\left(c \pm \sqrt{c^{2}-2 r}\right)-2 r\right\} \\
& =\frac{+2 h\left(c \pm \sqrt{c^{2}-2 r}\right)\left(-\frac{\lambda}{2}+\frac{\sqrt{\lambda^{2}-4 \mu}}{2} \times \tanh \left(\left(\sqrt{\lambda^{2}-4 \mu} / 2\right) \xi\right)\right)}{\left(h \lambda \pm \sqrt{c^{2}-2 r}\right)+2 h\left(-\frac{\lambda}{2}+\frac{\sqrt{\lambda^{2}-4 \mu}}{2} \times \tanh \left(\left(\sqrt{\lambda^{2}-4 \mu} / 2\right) \xi\right)\right)}
\end{aligned}
$$

Case 2: For $\lambda^{2}-4 \mu<0$,

$$
\begin{aligned}
& U_{2}(\xi) \\
& =\frac{+2 h\left(c \pm \sqrt{c^{2}-2 r}\right)\left(-\frac{\lambda}{2}+\frac{\sqrt{4 \mu-\lambda^{2}}}{2} \times \frac{-A \sin \left(\left(\sqrt{4 \mu-\lambda^{2}} / 2\right) \xi\right)+B \cos \left(\left(\sqrt{4 \mu-\lambda^{2}} / 2\right) \xi\right)}{A \cos \left(\left(\sqrt{4 \mu-\lambda^{2}} / 2\right) \xi\right)+B \sin \left(\left(\sqrt{4 \mu-\lambda^{2}} / 2\right) \xi\right)}\right)}{\left(h \lambda \pm \sqrt{c^{2}-2 r}\right)+2 h\left(-\frac{\lambda}{2}+\frac{\sqrt{4 \mu-\lambda^{2}}}{2} \times \frac{-A \sin \left(\left(\sqrt{4 \mu-\lambda^{2}} / 2\right) \xi\right)+B \cos \left(\left(\sqrt{4 \mu-\lambda^{2}} / 2\right) \xi\right)}{A \cos \left(\left(\sqrt{4 \mu-\lambda^{2}} / 2\right) \xi\right)+B \sin \left(\left(\sqrt{4 \mu-\lambda^{2}} / 2\right) \xi\right)}\right)}
\end{aligned}
$$

where $\xi=x-c t$.

Assign $A=0, B \neq 0$, then

$$
\begin{aligned}
& U_{2_{1}}(\xi) \\
& =\frac{\left\{(c+h \lambda)\left(c \pm \sqrt{c^{2}-2 r}\right)-2 r\right\}}{\left(h \lambda \pm \sqrt{c^{2}-2 r}\right)+2 h\left(-\frac{\lambda}{2}+\frac{\sqrt{4 \mu-\lambda^{2}}}{2} \times \cot \left(\left(\sqrt{4 \mu-\lambda^{2}} / 2\right) \xi\right)\right)}
\end{aligned}
$$

Fix $A \neq 0, B=0$, then

$$
=\frac{\left\{(c+h \lambda)\left(c \pm \sqrt{c^{2}-2 r}\right)-2 r\right\}+2 h\left(c \pm \sqrt{c^{2}-2 r}\right)\left(-\frac{\lambda}{2}+\frac{\sqrt{4 \mu-\lambda^{2}}}{2} \times\left(-\tan \left(\left(\sqrt{4 \mu-\lambda^{2}} / 2\right) \xi\right)\right)\right)}{\left(h \lambda \pm \sqrt{c^{2}-2 r}\right)+2 h\left(-\frac{\lambda}{2}+\frac{\sqrt{4 \mu-\lambda^{2}}}{2} \times\left(-\tan \left(\left(\sqrt{4 \mu-\lambda^{2}} / 2\right) \xi\right)\right)\right)}
$$

Case 3: When $\lambda^{2}-4 \mu=0$,

$$
=\frac{\left\{(c+h \lambda)\left(c \pm \sqrt{c^{2}-2 r}\right)-2 r\right\}+2 h\left(c \pm \sqrt{c^{2}-2 r}\right)\left(-\frac{\lambda}{2}+\frac{B}{A+B \xi}\right)}{\left(h \lambda \pm \sqrt{c^{2}-2 r}\right)+2 h\left(-\frac{\lambda}{2}+\frac{B}{A+B \xi}\right)}
$$


where $\xi=x-c t$.

Put $A=0, B \neq 0$,

$$
=\frac{\left\{(c+h \lambda)\left(c \pm \sqrt{c^{2}-2 r}\right)-2 r\right\}+2 h\left(c \pm \sqrt{c^{2}-2 r}\right)\left(-\frac{\lambda}{2}+\frac{1}{\xi}\right)}{\left(h \lambda \pm \sqrt{c^{2}-2 r}\right)+2 h\left(-\frac{\lambda}{2}+\frac{1}{\xi}\right)}
$$

Choose $A \neq 0, B=0$, then

$$
=\frac{\left\{(c+h \lambda)\left(c \pm \sqrt{c^{2}-2 r}\right)-2 r\right\}+2 h\left(c \pm \sqrt{c^{2}-2 r}\right)\left(-\frac{\lambda}{2}\right)}{\left(h \lambda \pm \sqrt{c^{2}-2 r}\right)+2 h\left(-\frac{\lambda}{2}\right)}
$$

\section{Results, discussion and graphical representations}

To analyse the problems clearly and to describe the solutions of the phenomena, a graphical representation of the solutions can be an effective tool to brief the commentaries. On account of this, we provide different types of physical appearances of the solutions bearing the actual form of solitary waves (Fig. 1Fig. 6). The plots are of kink shape soliton, cuspone, periodic solutions etc. Fig. 1 stands for kink type soliton of solution (17) for $\lambda=3, \mu=2, c=1, a=1, b=1, x=0$ in the interval $-10 \leq y \leq 10$ and $-10 \leq t \leq 10$. Fig. 2 characterizes cuspon which is depicted for solution (17) for $\lambda=3, \mu=3, c=-1$, $a=1, b=1, x=0$ within $-10 \leq y \leq 10$ and $-10 \leq t \leq$ 10. Fig. 3 indicates periodic soliton generated from the solution (17) for $\lambda=3, \mu=3, c=1, a=1, b=1, x=0$ in $-10 \leq$ $y \leq 10$ and $-10 \leq t \leq 10$. Fig. 4 indicates cuspon plotted for solution (17) for $\lambda=3, \mu=2, c=-1, a=1, b=1, x=0$ for the interval $-10 \leq y \leq 10$ and $-10 \leq t \leq 10$. Fig. 5 designates singular kink type soliton emerged from the solution (29) for $\lambda=4, \mu=2, c=-5, h=-5, r=1$ within $-10 \leq$ $x \leq 10$ and $-10 \leq t \leq 10$. Fig. 6 represents kink soliton from the solution (29) for $\lambda=7, \mu=3, c=-5, h=-5, r=-1$ in the intervals $-10 \leq x \leq 10$ and $-10 \leq t \leq 10$. Fig. 7 designates cuspon emerged from solution (29) for $\lambda=3, \mu=$ $4, c=5, h=-1, r=-1$ for $-10 \leq x \leq 10$ and $-10 \leq t \leq$ 10. Fig. 8 designates singular kink type soliton emerged from the solution (29) for $\lambda=-3, \mu=0, c=5, h=-3, r=1$ in the interval $-10 \leq x \leq 10$ and $-10 \leq t \leq 10$.

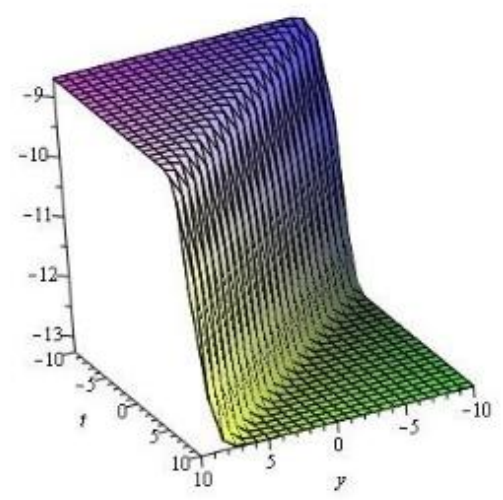

Fig. 1

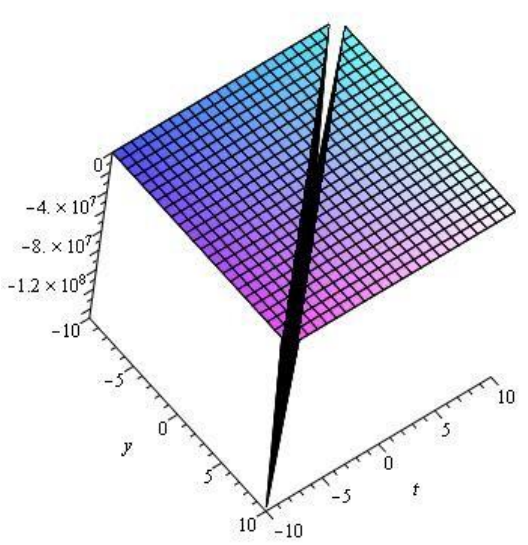

Fig. 2

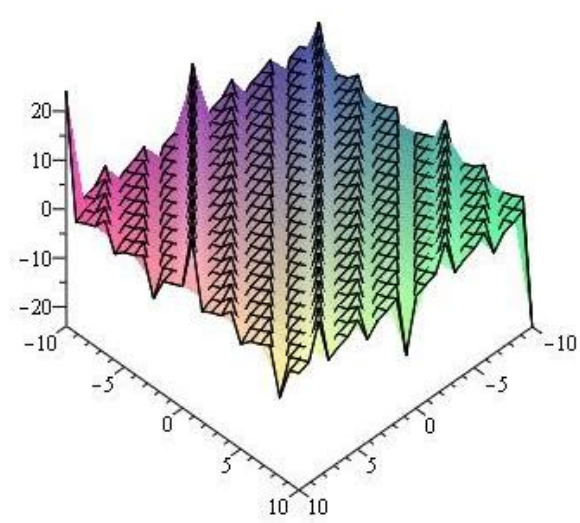

Fig. 3

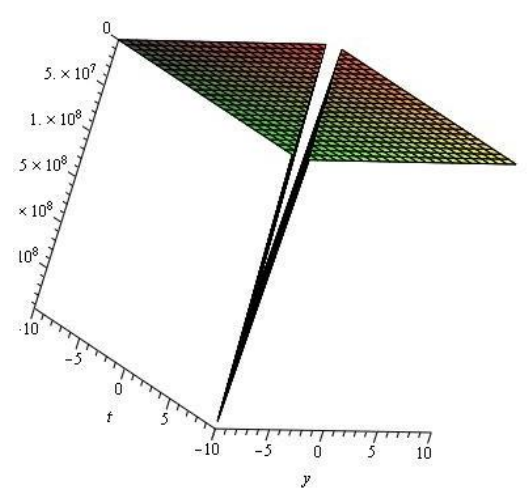

Fig. 4

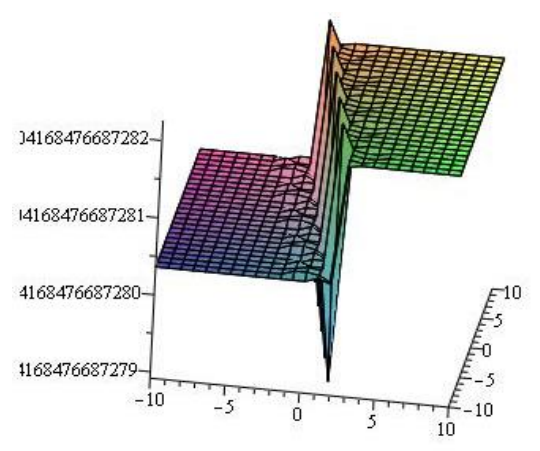

Fig. 5 


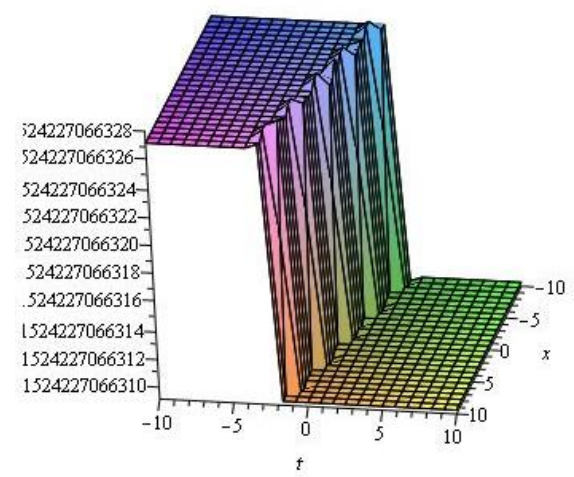

Fig. 6

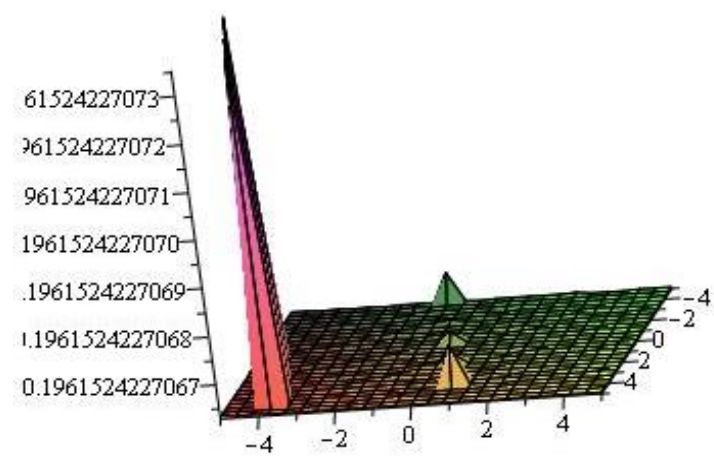

Fig. 7

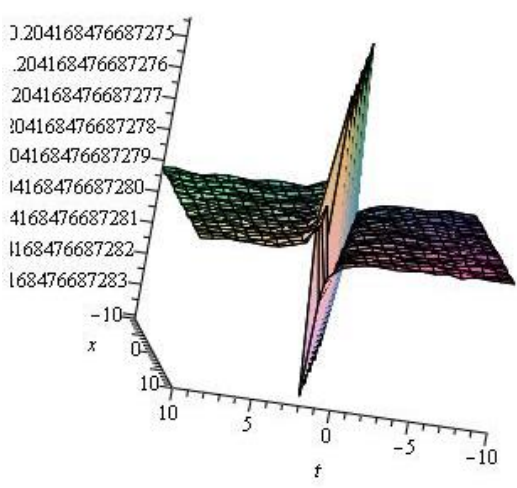

Fig. 8

\section{Conclusions}

The determination of this article was to investigate closed form analytic solutions to the $\mathrm{cKG}$ and $\mathrm{BO}$ equations by employing the rational $\left(\mathrm{G}^{\wedge} / \mathrm{G}\right)$-expansion method. Consequently, different and advanced travelling wave solutions to the considered equations have successfully been furnished, on comparison to other methods available in the literature. Our derived solutions might effectively be helpful to depict the interior behaviors of internal mechanisms of nature world like describing shallow water waves, acoustic waves etc. The gained hyperbolic, trigonometric and rational function solutions together with the physical appearances show the efficiency and the reliability of our employed method which might be used in further research works to find fresh and further general solutions of any other NLPDEs in different fields.

\section{References}

[1] Wazwaz, A.M., 2002. Partial differential equations: methods and applications. AA Balkema.
[2] Helal, M.A. and Mehanna, M.S., 2006. A comparison between two different methods for solving KdV-Burgers equation. Chaos, Solitons \& Fractals, 28(2), pp.320-326.

[3] Parkes, E.J. and Duffy, B.R., 1996. An automated tanhfunction method for finding solitary wave solutions to nonlinear evolution equations. Computer Physics Communications, 98(3), pp.288-300.

[4] Abdou, M.A., 2007. The extended tanh method and its applications for solving nonlinear physical models. Applied mathematics and computation, 190(1), pp.988-996.

[5] Kudryashov, N.A., 1990. Exact solutions of the generalized Kuramoto-Sivashinsky equation. Physics Letters A, 147(56), pp.287-291.

[6] Kudryashov, N.A., 2005. Simplest equation method to look for exact solutions of nonlinear differential equations. Chaos, Solitons \& Fractals, 24(5), pp.1217-1231.

[7] Xu, G., 2006. An elliptic equation method and its applications in nonlinear evolution equations. Chaos, Solitons \& Fractals, 29(4), pp.942-947.

[8] Parkes, E.J., Duffy, B.R. and Abbott, P.C., 2002. The Jacobi elliptic-function method for finding periodic-wave solutions to nonlinear evolution equations. Physics Letters A, 295 (56), pp.280-286.

[9] He, J.H. and Wu, X.H., 2006. Exp-function method for nonlinear wave equations. Chaos, Solitons \& Fractals, 30(3), pp.700-708.

[10] Naher, H., Abdullah, F.A. and Akbar, M.A., 2012. New traveling wave solutions of the higher dimensional nonlinear partial differential equation by the Exp-function method. Journal of Applied Mathematics, 2012.

[11] Khan, K., Akbar, M. A. and Alam, M. N., 2013. "Travelling wave solutions of the nonlinear Drinfel'd-Shokolov-Wilson equation and modified Benjamin-Bona-Mahony equations," Journal of the Egyptian Mathematical Society, 21, pp. 233240.

[12] Islam, M.H., Khan, K., Akbar, M.A. and Salam, M.A., 2014. Exact traveling wave solutions of modified KdV-ZakharovKuznetsov equation and viscous Burgers equation. SpringerPlus, 3(1), pp.1-9.

[13] Wang, M., Li, X. and Zhang, J., 2008. The (G' G)-expansion method and travelling wave solutions of nonlinear evolution equations in mathematical physics. Physics Letters A, 372(4), pp.417-423.

[14] Ebadi, G. and Biswas, A., 2011. The $\mathrm{G}^{\prime} \mathrm{G}$ method and topological soliton solution of the $\mathrm{K}(\mathrm{m}, \mathrm{n})$ equation. Communications in Nonlinear Science and Numerical Simulation, 16(6), pp.2377-2382.

[15] Biswas, A., 2009. Solitary wave solution for the generalized Kawahara equation. Applied Mathematics Letters, 22(2), pp.208-210.

[16] Biswas, A., Petković, M.D. and Milović, D., 2010. Topological and non-topological exact soliton solution of the power law KdV equation. Communications in Nonlinear Science and Numerical Simulation, 15(11), pp.3263-3269.

[17] Chun, C. and Sakthivel, R., 2010. Homotopy perturbation technique for solving two-point boundary value problemscomparison with other methods. Computer Physics Communications, 181(6), pp.1021-1024.

[18] Sakthivel, R., Chun, C. and Lee, J., 2010. New travelling wave solutions of Burgers equation with finite transport memory. Zeitschrift für Naturforschung A, 65(8-9), pp.633640. 
[19] He, J.H. and Wu, X.H., 2006. Exp-function method for nonlinear wave equations. Chaos, Solitons \& Fractals, 30(3), pp.700-708.

[20] Ma, W.X., Huang, T. and Zhang, Y., 2010. A multiple expfunction method for nonlinear differential equations and its application. Physica Scripta, 82(6), p.065003.

[21] Zheng, B., 2011. A new Bernoulli sub-ODE method for constructing traveling wave solutions for two nonlinear equations with any order. UPB Sci. Bull., Series A, 73(3), pp.85-94.

[22] Zheng, B., 2012. Soling a nonlinear evolution equation by a proposed Bernoulli sub-ODE method. In Int. Conf. Image Vis. Comput.

[23] Abbasbandy, S., 2010. Homotopy analysis method for the Kawahara equation. Nonlinear Analysis: Real World Applications, 11(1), pp.307-312.

[24] Molabahrami, A. and Khani, F., 2009. The homotopy analysis method to solve the Burgers-Huxley equation. Nonlinear Analysis: Real World Applications, 10(2), pp.589600.

[25] Molliq, Y., Noorani, M.S.M. and Hashim, I., 2009. Variational iteration method for fractional heat-and wavelike equations. Nonlinear Analysis: Real World Applications, 10(3), pp.1854-1869.

[26] Mohyud-Din, S.T., Noor, M.A. and Noor, K.I., 2009. Modified Variational Iteration Method for Solving SineGordon Equations, World Applied Sciences Journal, 6(7), pp. 999-1004.

[27] Lee, J. and Sakthivel, R., 2014. Exact travelling wave solutions of a variety of Boussinesq-like equations.," Chinese Journal of Physics, 52(3), pp. 939-957.

[28] Ganji, D.D., 2006. The application of He's homotopy perturbation method to nonlinear equations arising in heat transfer. Physics Letters A, 355(4-5), pp.337-341.

[29] Ganji, D.D., Afrouzi, G.A. and Talarposhti, R.A., 2007. Application of variational iteration method and homotopyperturbation method for nonlinear heat diffusion and heat transfer equations. Physics Letters A, 368(6), pp.450-457.

[30] Wang, M., 1995. Solitary wave solutions for variant Boussinesq equations. Physics letters A, 199(3-4), pp.169172.

[31] Ablowitz, M.J., Ablowitz, M.A., Clarkson, P.A. and Clarkson, P.A., 1991. Solitons, nonlinear evolution equations and inverse scattering (Vol. 149). Cambridge University Press, UK.

[32] Rogers, C. and Shadwick, W.F., 1982. Bäcklund transformations and their applications. Academic Press, New York.

[33] Jianming, L., Jie, D. and Wenjun, Y., 2011, January. Bäcklund transformation and new exact solutions of the Sharma-Tasso-Olver equation. In Abstract and Applied Analysis (Vol. 2011). Hindawi.

[34] Seadawy, A.R., 2015. Fractional solitary wave solutions of the nonlinear higher-order extended $\mathrm{KdV}$ equation in a stratified shear flow: Part I. Computers \& Mathematics with Applications, 70(4), pp.345-352.

[35] Seadawy, A.R., 2016. Three-dimensional nonlinear modified Zakharov-Kuznetsov equation of ion-acoustic waves in a magnetized plasma. Computers \& Mathematics with Applications, 71(1), pp.201-212.

[36] Arshad, M., Seadawy, A., Lu, D. and Wang, J., 2016. Travelling wave solutions of generalized coupled ZakharovKuznetsov and dispersive long wave equations. Results in Physics, 6, pp.1136-1145.

[37] Seadawy, A.R., El-Kalaawy, O.H. and Aldenari, R.B., 2016. Water wave solutions of Zufiria's higher-order Boussinesq type equations and its stability. Applied Mathematics and Computation, 280, pp.57-71.

[38] Islam, M., Akbar, M.A. and Azad, A.K., 2015. A Rational $\left(\mathrm{G}^{\prime} / \mathrm{G}\right)$-expansion method and its application to the modified KdV-Burgers equation and the $(2+1)$-dimensional Boussinesq equationn. Nonlinear Studies, 22(4).

[39] Islam, M.T., Akbar, M.A. and Azad, M.A.K., 2017. Multiple closed form wave solutions to the $\mathrm{KdV}$ and modified $\mathrm{KdV}$ equations through the rational $\left(\mathrm{G}^{\prime} / \mathrm{G}\right)$-expansion method. Journal of the Association of Arab Universities for Basic and Applied Sciences, 24, pp.160-168.

[40] Islam, M.T., Ali, M.A. and Hasan, M.R., Closed Form Wave Solutions to the Nonlinear Partial Differential Equations via the Rational)/'(GG-Expansion Method. International Journal for Research in Applied Science \& Engineering Technology, 6(2), pp 1519-1525.

[41] Akbar, M.A., Ali, N.H.M. and Islam, M.T., 2019. Multiple closed form solutions to some fractional order nonlinear evolution equations in physics and plasma physics. AIMS Mathematics, 4(3), pp.397-411. 